\title{
Enunciación
}

http://revistas.udistrital.edu.co/ojs/index.php/enunc

DOI: http://dx.doi.org/10.14483/udistrital.jour.enunc.2015.1.a01

enunciación

Artículo DE INVESTIGACIÓN

\section{Construcción del concepto de tecnología en una red virtual de aprendizaje}

\section{Construction of the concept of technology in a virtual learning network}

\author{
Ruth Molina Vásquez ${ }^{1}$
}

Para citar este artículo: Vásquez, R. M. (2015). Construcción del concepto de tecnología en una red virtual de aprendizaje. Enunciación, 20(1), pp. 10-25.

Recibido: 30-abril-2015 / Aprobado: 25-mayo-2015

\section{Resumen}

Este artículo presenta los resultados de la tesis doctoral titulada "Construcción del concepto de tecnología en una red virtual de aprendizaje", realizada en el Doctorado Interinstitucional en Educación (DIE) de la Universidad Distrital Francisco José de Caldas. El objetivo de esta investigación es caracterizar el proceso que realizan estudiantes de séptimo grado al construir el concepto de tecnología cuando interactúan en una red virtual de aprendizaje. A partir de elementos teóricos desde las redes de conocimiento en los procesos educativos, los enfoques sobre tecnología y los procesos de conceptualización, se diseña y desarrolla la Red virtual de aprendizaje en tecnología (Revatec). Con base en el desarrollo de las actividades propuestas allí, se realiza desde la metodología de teoría fundamentada un análisis de las interacciones y el proceso de construcción del concepto de tecnología que realizan estudiantes de séptimo grado que participan en esta red. Los resultados muestran que el concepto de tecnología se construye desde cuatro categorías: 1) artefactos, materiales e instrumentos, 2) aspectos sociales y culturales, 3) sistemas, conocimientos y procesos, y 4) aplicación de la ciencia. Esta construcción se realiza a partir del entrelazamiento de las cuatro categorías enunciadas, de manera multidimensional, y desde artefactos de carácter tecnológico, simbólico, cultural y científico, que buscan proporcionar bienestar, calidad de vida, satisfacer las necesidades, vacíos de conocimiento, de comprensión y transformación del mundo, a partir de la producción de nuevos procesos, sistemas y contextos sociales y culturales.

Palabras clave: red virtual de aprendizaje, enfoques de tecnología, construcción conceptual, teoría fundamentada.

\begin{abstract}
This paper presents the research results of the $\mathrm{PhD}$ thesis entitled "Construction of the concept of technology in a virtual learning network". The objective of this research is to characterize the process that made seventh graders build the concept of technology as they interact in a virtual learning network. Supported by theoretical elements from networks of knowledge in education, conceptions of technology and processes of conceptualization, it designs and develops virtual learning Revatec Network technology. Based on the development of proposed activities, from the grounded theory methodology, the interactions and the construction process of the concept of technology that seventh grader students who participate in this network make is analyzed, where instrumental, cognitive, systemic and cultural categories are shown.
\end{abstract}

Keywords: Virtual learning network, technology approaches, conceptual construction, grounded theory.

$1 \quad$ Magíster en Tecnologías de la Información Aplicadas a la Educación, candidata a doctora en Educación. Docente de planta de la Facultad de Ciencias y Educación, Universidad Distrital Francisco José de Caldas. Correo electrónico: rmolinav@udistrital.edu.co 


\section{INTRODUCCIÓN}

La adopción de diferentes tipos de tecnologías ha cambiado los aspectos formales del desarrollo de las actividades tradicionales en el aula de clase al dar paso a la digitalización de materiales, al acceso inmediato a la información y a nuevas formas de comunicación asíncrona entre estudiantes y profesores. Pero más allá del hecho de cambiar los medios utilizados, la incorporación de las tecnologías de la información y la comunicación ha brindado posibilidades de encuentro social entre los jóvenes, de acceso a la información, a la manera como se interrelacionan y como construyen sus identidades en las redes. Ahora bien, el auge que las redes han tenido entre los jóvenes, particularmente las de tipo social, conlleva a preguntarse si el uso de redes en los procesos educativos tendrá el mismo impacto y cuál sería su incidencia en los procesos de enseñanza y, particularmente, en la forma como se aprende. Más allá de esto, y dado que en la actualidad este tipo de tecnologías se encuentran inmersas en la vida diaria, surge la inquietud acerca de cuál es el concepto que tienen los jóvenes sobre la tecnología.

Los aspectos de interés que suscita este estudio son dos: por un lado, la formación del concepto de tecnología y, por otro, el uso de las redes virtuales de aprendizaje (RVA) como mediadoras en su construcción. Por ello, el problema de investigación planteado se concreta en la siguiente pregunta: ¿Cuáles son las características del proceso de construcción del concepto de tecnología que realizan los estudiantes que participan en una red virtual de aprendizaje? A partir de este interrogante se plantean las siguientes subpreguntas: ¿Cuáles son las características pedagógicas, tecnológicas, comunicativas y de contenido, de una red virtual de aprendizaje que busca facilitar la construcción del concepto de tecnología en los estudiantes de educación básica?, ¿qué tipo de transformaciones se presentan en el concepto de tecnología durante la interacción en la red virtual de aprendizaje? y ¿cuáles son las características de la interacción entre los participantes de la red en ese proceso de construcción?

El objetivo general de esta investigación es determinar las características del proceso de construcción del concepto de tecnología que realizan estudiantes de séptimo grado de educación básica a partir de su interacción en una red virtual de aprendizaje. Los objetivos específicos se orientan a: 1) Adoptar un enfoque pedagógico, didáctico, tecnológico, administrativo y de contenidos para realizar el diseño, desarrollo e implementación de la red virtual de aprendizaje que facilite la construcción del concepto de tecnología. 2) Analizar los cambios presentados por los estudiantes de séptimo grado de educación básica con respecto al concepto de tecnología durante la interacción en la red virtual de aprendizaje. 3) Caracterizar el proceso de interacción realizado por los estudiantes participantes de la red virtual de aprendizaje.

Este proceso de investigación busca contribuir a determinar las características de la construcción de conceptos en el área de tecnología y aportar al cuerpo de conocimientos de esta área reciente del currículo colombiano, así como esclarecer la utilidad mediadora de las tecnologías de información y comunicación (TIC), particularmente de las RVA. Indagar sobre el proceso de construcción del concepto de tecnología lleva a preguntarse por la forma como este se configura en el contexto escolar a la luz de los aspectos que lo componen (Cupani, 2006; Cajas, 2001), y cómo se utiliza en la mediación del aprendizaje. Inicialmente es necesario analizar el referente contextual del área de tecnología e informática en la educación básica, secundaria y media en el país, en donde se encuentra que es relativamente reciente, que el $70 \%$ de los docentes que trabajan en el área no han sido formados en ella (Briceño, Lugo, Molina, y Munevar, 2006) y que su orientación es fundamentalmente técnica, lo cual redunda en una mirada operativa que se refleja en la formación inicial de los docentes y en la escasa reflexión sobre el sentido de la tecnología (Rueda, 2006). 
La discusión sobre la formación en el concepto de tecnología en el ámbito internacional no es un elemento común y tan solo se trata tangencialmente en la formulación de estándares. Uno de los más difundidos ha sido el de la International Society for Technology in Education (Iste), que define las competencias en educación en tecnología para la próxima generación, incluyendo la creación e innovación, comunicación y colaboración, búsqueda y manejo de información, pensamiento crítico, solución de problemas y toma de decisiones, ciudadanía digital y operaciones y concepto de tecnología (Iste, 2007). A nivel nacional, la tecnología e informática es un área de conocimiento obligatoria en la educación básica y media desde 1994, con la Ley General de Educación; solo catorce años más tarde se publican las "Orientaciones generales para la educación en tecnología" (Ministerio de Educación Nacional, 2008), en donde se incluyen cuatro componentes para la formación: naturaleza de la tecnología, apropiación y uso de la tecnología, solución de problemas con tecnología y tecnología y sociedad. El primero de ellos incluye la competencia conceptual en el grupo de grados de sexto a séptimo, así: "Reconozco principios y conceptos propios de la tecnología, así como momentos de la historia que le han permitido al hombre transformar el entorno para resolver problemas y satisfacer necesidades" (Ministerio de Educación Nacional, 2008).

Algunas investigaciones se han ocupado de estudiar la conformación de RVA con docentes de educación básica y media (Molina y Briceño, 2010; Maldonado y Serrano, 2008), las estrategias de participación (Molina y Briceño, 2009) y su uso en la formación e investigación con maestros de matemáticas (Bonilla, Molina, Martínez y Narváez, 2011; Angulo, Garzón, Mejía, Obando y Posada, 2007). Los resultados permiten caracterizar su conformación, el proceso de construcción individual de conocimiento, el tipo de participación que realizan los docentes que se encuentran registrados en ellas, así como sus procesos de consolidación.
Ninguna de estas experiencias se desarrolla con jóvenes en edad escolar, lo que Ileva a preguntarse sobre la manera como una RVA puede ser un elemento de mediación pedagógica en el proceso de construcción conceptual en el contexto escolar, si las dinámicas de aprendizaje de los niños serán similares a las que han presentado los docentes y cuáles serán las características del proceso de construcción en torno del concepto de tecnología.

Este estudio considera las RVA como mediadoras en la construcción de conocimiento, a partir de elementos teóricos basados en la gestión de conocimiento (Hakkarainen, Palonen, Paavola y Lehtinen, 2004), concebida desde procesos de construcción, prueba, representación, debate y uso de conocimientos, frente al cual se puede tener al menos tres enfoques: la adquisición de conocimiento, centrado en los procesos de transmisión y representación de contenidos; la construcción desde la participación y el aprendizaje colaborativo con objetivos comunes; y la creación consolidada en comunidades o en redes. Las RVA son un tipo de red de conocimiento, por tanto se fundamentan en una idea de comunidad (Benassini, 2003), en la que persiste la comunicación y el propósito implícito de desarrollar un proyecto grupal, lo cual es posible gracias a las redes telemáticas que facilitan la comunicación interactiva y que permiten a sus miembros compartir información e innovación; tienen lugar en ambientes formales de enseñanza aprendizaje y se apoyan en el acceso a la información y a procesos de comunicación, en un espacio en la red cuyo objeto es potenciar el aprendizaje a partir de actividades como solucionar problemas, desarrollar estructuras conceptuales, realizar construcción de conocimiento. Se caracterizan por la interacción y la proximidad psicológica entre sus miembros (Harasim, Starr, Murria y Teles, 2000), aprendizaje colaborativo (Johnson y Johnson, 1998), y conocimiento compartido (Dillenbourg, 1996).

En diversos procesos de investigación se ha encontrado que uno de los aspectos más relevantes de las RVA es la interacción (Maldonado y 
Serrano, 2008; Molina y Briceño, 2009), entendida como la acción que se ejerce recíprocamente entre dos o más objetos, agentes, fuerzas, funciones, etc. Este concepto se ha trabajado desde diferentes áreas de conocimiento, incluyendo las ciencias de la comunicación. Sin embargo, este estudio adopta el concepto de interacción desde la perspectiva de la educación a distancia, donde se entiende como la comunicación bidireccional y dialógica entre estudiante y docente (Bossolasco, 2010; García, 2001; Barbera, Badia y Mominó, 2001), como un tipo de conversación guiada de forma didáctica que se sostiene de dos formas: real cuando se presenta de manera directa entre docente-estudiante y simulada cuando se sostiene a través de un material didáctico.

Esta postura coincide con la propuesta de Jonnasen, Davidson, Collins, Campbell y Haag (2007), quienes señalan dos dimensiones de la educación a distancia: una negociación social entre los participantes y una negociación interna entre el estudiante y el contenido. En la tercera etapa de la educación a distancia estos tipos de interacción están mediados por el computador y por tanto adquieren características diferentes a las que se observaban con el uso de medios masivos (García, 2001) y empiezan a entenderse como el diálogo que se establece entre el hombre y el entorno digital (De Kerckhove, 1999), a partir de procesos de diseño de interfaz (Scolari, 2004), y también como la comunicación que se presenta entre los actores del proceso educativo, mediada por las TIC (Hillman, Wills y Gunawardena, 1994; Rengil, 2002).

Este estudio asume la interacción desde una perspectiva integral compuesta por tres aspectos: la interacción con el entorno digital, desde una metáfora de diseño (la interfaz); la interacción con los contenidos, entendidos estos como mediadores de conocimiento; y la interacción social entre los participantes de la RVA. Las características descritas apoyan el desarrollo de procesos de carácter individual de asimilación, reestructuración o cambio de las estructuras cognitivas del sujeto, que le permiten resignificar la nueva información que se le presenta (Ausubel, Novak y Henesian, 1983); y otra de carácter social desde la interacción social (Vygotski, 1934; Dillenbourg, 1996), que favorecen aprendizajes de carácter colaborativo (Johnson y Johnson, 1998; Palloff y Pratt, 2005; Gros, 2004).

Con respecto al concepto de tecnología, se observan dos tendencias (Mitcham, 1998), la primera de ellas desde una mirada ingenieril, enraizada en aspectos como la concreción material y extensión de órganos (Rapp, 1981), el análisis de las máquinas (Mumford, 1971), el afán de poder y dominio de la naturaleza (Hronzsky, 2001), desde la generaciònd e actos de creaciòn (Desauer, citado por Mitcham, 1998), que tiene la capacidad de responder a nuevas demandas. La segunda tendencia, de carácter humanista, se ocupa de analizar las visiones instrumentalistas y antropológicas de la técnica como complementarias (Heidegger, 1994), de concebir la técnica ligada a lo que significa ser humano y los procesos de dominación (Rapp, 1981), en estrecha relación con la configuración cultural de las sociedades (Quintanilla, 1993-1994) y con participación política (Winner, 1985). Desde la sociología de la tecnología se plantea la inquietud por las relaciones que se establecen entre tecnología y sociedad, a la cual se da respuesta desde las perspectivas de la teoría del actor red (Latour, 2005), desde el modelo sistémico (Hughes, 1983) y de construcción social de la tecnología (Pinch y Bijker, 1984; Pinch, 1997).

Esta última perspectiva entiende los artefactos tecnológicos como construcciones sociales y presupone que hay muchas soluciones diferentes para los problemas técnicos y diversos caminos para llegar a ellas, lo cual significa romper con la visión lineal de la innovación, por un lado, y, por otro, negar que la tecnología se desarrolle de manera independiente de la sociedad (Osorio, 2007). Esta postura deja atrás cualquier supuesto de neutralidad (Broncano, 2000); asume la comprensión de la tecnología desde rangos culturales interrelacionados de tipo simbólico, práctico y axiológico (Quintanilla, 1993-1994), a partir del análisis del discurso tecnológico y sus prácticas, en un sistema 
complejo, compuesto por materiales, agentes humanos y relaciones de transformación, como forma de vida y construcción del mundo (Winner, 1985).

Entre los enfoques más comunes del concepto de tecnología se encuentran (Osorio, 2003): 1) artefactual, en donde la tecnología se entiende como herramientas o máquinas; 2) cognitivo, que entiende la tecnología como una aplicación de la ciencia; 3) sistémico, que concibe la tecnología como un sistema complejo. De otra parte, se empiezan a encontrar reflexiones en torno a la tecnología, referidas a dominios simbólicos, organizativos y técnicos que impactan la construcción cultural (Cole, 1999), diluyendo las fronteras disciplinares (Medina, 2003) y orquestada en las interconexiones en el ciberespacio, en donde las personas se concentran y se interrelacionan, cambian las formas tradicionales de encuentro con el otro, de comunicación, de constitución de sujeto y la manera como se construyen los esquemas compartidos intersubjetivamente., en lo que se ha denominado cibercultura (Lévy, 2007). Estas miradas diversas fundamentan el proceso histórico como se ha concebido el concepto de tecnología desde diferentes orillas teóricas.

Los procesos de construcción conceptual, por su parte, se fundamentan teóricamente en: la epistemología genética o estructuralismo (Piaget, 1972), que destaca las etapas de desarrollo basadas en nociones, conceptos y categorías, por las que se transita gracias a procesos de equilibrio y desequilibrio; el aprendizaje significativo (Ausubel, Novak y Henesian, 1983), desde la formación de conceptos y los procesos de asimilación; los procesos conceptuales desde la construcción de conglomerados, colecciones y conceptos, y la generación de procesos sociales desde la interacción (Vygotski, 1934), en donde se destaca el concepto de zona de desarrollo próximo. Estos elementos se configuran como el fundamento teórico que da pie para el diseño tanto de los contenidos como de las actividades que se proponen en la Revatec y que se presentan en el siguiente apartado.

\section{METODOLOGÍA DE INVESTIGACIÓN}

El proceso metodológico del estudio se realiza en dos etapas: una primera etapa de diseño de la Red virtual de aprendizaje en tecnología (Revatec) y una segunda etapa de implementación de esta red. El diseño de la Revatec se basa en el modelo de Addie (Williams, 2007), que cuenta con varias etapas: análisis, diseño, desarrollo, implementación y evaluación. La etapa de análisis parte de revisar algunas redes virtuales de aprendizaje y sociales especializadas para niños y adolescentes, consultar sus expectativas frente a lo que desean ver en internet, su uso del computador e internet y sus preferencias de diseño (Creafuture, 2010); se presentan los resultados de entrevistas a adolescentes del contexto local, en las que se indaga por los usos de internet, del computador, de redes virtuales de aprendizaje y sus preferencias de diseño de páginas, con el fin de caracterizar a los usuarios de la red.

En la etapa de diseño se consideran cuatro componentes: pedagógico, comunicativo, técnico-administrativo y de contenidos (Unigarro et al., 2003). El diseño pedagógico de la red se fundamenta en los aprendizajes significativos y colaborativos a partir de los cuales se formulan estrategias metodológicas y didácticas planteadas por autores como Ausubel, Novak y Henesian (1983), Piaget (1972), Vygotski (1934), que se desarrollan en el planteamiento de cuatro actividades de aprendizaje. El diseño comunicativo se fundamenta en procesos de interacción con la interfaz, con los contenidos y la interacción social, que se recrea en una metáfora espacial o tridimensional, que toma la forma en el entorno de una oficina de detectives, en donde se privilegia la imagen de espacios en los que se realizan diversas acciones. El diseño técnico y administrativo se realiza en un ambiente Moodle, incorporando el manejo de lenguajes de Java, HTML y PHP, cuya arquitectura se divide en módulos de presentación, exploración, actividades de aprendizaje, consulta, interacción e 
información general'2. El diseño de contenidos se fundamenta en los componentes incluidos en las "Orientaciones generales para la educación en tecnología" (Ministerio de Educación Nacional, 2008): naturaleza de la tecnología, apropiación y uso de la tecnología, solución de problemas con tecnología y tecnología y sociedad.

Estos elementos permiten desarrollar una estrategia basada en el planteamiento del reto de descubrir la identidad de un personaje misterioso, a partir de la realización de actividades de las siguientes unidades: "Tras la búsqueda del personaje", "Problemas que resuelve el personaje misterioso", "De la historia a las pistas actuales" e "Impacto del personaje". Finalmente, la etapa de validación del desarrollo de la red es realizada, con base en la estrategia Delphi, por parte de tres expertos en tecnología, educación en tecnología y redes virtuales de aprendizaje, quienes utilizan el "Formato de catalogación y evaluación de software educativo" (Marqués, 2001) para evaluar aspectos funcionales, técnicos, estéticos y pedagógicos de la Revatec, además de entrevistas a participantes de la red. Con base en los resultados de esta evaluación, se realizan ajustes en aspectos gráficos y de extensión de contenidos.

En la aplicación de la Revatec, el estudio acude a una perspectiva metodológica de investigación desde la teoría fundamentada, debido a que se busca que las características del proceso de construcción del concepto de tecnología emerjan en las interacciones de los participantes y no a partir de categorías de análisis preconcebidas, tal como se plantea en este tipo de metodología (Strauss y Corbin, 2002; Hernández, 2008; Inciarte, 2011). Esto implica que los elementos teóricos planteados en el estudio se configuran como fundamento del diseño de la Revatec, mas no se implementan como elementos de análisis de los resultados.

2 El desarrollo de la Revatec se encuentra en http://ciencias.udistrital. edu.co/avirtual/course/view.php?id=55
La teoría fundamentada es una metodología de investigación genérica de los estudios cualitativos que se orienta a explicar en un nivel conceptual, de acción, de interacción o en un área específica los datos analizados de manera sistemática (Hernández, 2008; Creswell, 2005; Mertens, 2005). El proceso metodológico de análisis de datos desde la teoría fundamentada (Strauss, 1987; Charmaz, 2000; Strauss y Corbin, 2002; Clarke, 2005; Inciarte, 2011) se realiza a partir de la aplicación de un método de comparación constante de las situaciones, objetos, incidentes que se revelan en los datos, "en busca de similitudes y diferencias... a fin de clasificarlas" (Strauss y Corbin, 2002, p. 103). En una primera instancia, el resultado de este proceso es la determinación de conceptos, entendidos como "un fenómeno al que se le ha puesto una etiqueta. Se trata de una representación abstracta de un acontecimiento, objeto, acción/interacción que un investigador identifica como significativa en los datos" (Strauss y Corbin, 2002, p. 112). Para este estudio se configuran desde las expresiones que realizan los participantes en interacción en la Revatec. En una segunda instancia se realiza una comparación entre estos conceptos extraídos "para buscar conceptos similares y diferentes, a los que se les puedan sacar las posibles propiedades y dimensiones" (Strauss y Corbin, 2002, p. 103). El resultado de este proceso son categorías más abstractas en las que se pueden agrupar los conceptos, a fin de extraer posibles propiedades y elementos comunes que, en este estudio, se configuran como las características de la construcción del concepto de tecnología que realizan los participantes.

Este método de comparación constante no incluye la comparación entre aspectos teóricos previamente consultados y los aspectos que revelan los datos, sino que se orienta a realizar un microanálisis de los datos obtenidos en los registros generados, en este caso en las herramientas asíncronas y sincrónicas utilizadas en la Revatec y en las cuales participan los niños y niñas de este estudio. Las expresiones analizadas se encuentran 
en los protocolos de las herramientas colaborativas como foros y wiki y las herramientas comunicativas como mensajes, chat y videos, incluidas en las actividades de aprendizaje de las cuatro unidades de la Revatec: Unidad 1. Tras la búsqueda de nuestro personaje; Unidad 2. Problemas que resuelve el personaje misterioso; Unidad 3. De la historia a las pistas actuales; y Unidad 4. Impacto de nuestro personaje.

En estos protocolos se identifican un total de 1.257 expresiones, es decir oraciones con sentido completo (en este estudio son desechadas aquellas expresiones que no tienen sentido en sí mismas), las cuales son analizadas utilizando la plataforma de AtlasTi, desde donde se codifican en conceptos y se agrupan en familias de categorías que son graficadas en redes de relaciones. Como se observa en la Figura 1, el microanálisis parte de realizar un análisis línea por línea tanto de los protocolos de interacción de los estudiantes como de las entrevistas, con el objeto de haIlar elementos comunes que permitan agruparlos en unos códigos y descubrir las relaciones entre ellos. Luego se realiza una codificación abierta, en donde se adjudican conceptos que recogen la esencia de cada una de las expresiones de los participantes, se agrupan en conceptos y se agregan comentarios y memorandos que ayudan a perfilar conceptos iniciales presentes en los datos.

Posteriormente se realiza una codificación axial, en donde se agrupan los conceptos en categorías y se establecen sus características y relaciones, seguido de una codificación selectiva en que se busca una categoría central que explique el proceso de conceptualización utilizado, a partir de la cual se procede a plantear una teoría sustantiva que determine las características de la interacción entre los participantes y la manera como construyen el concepto de tecnología.

En el estudio participan 169 niños y niñas con edades entre 12 y 15 años, con conocimientos básicos de manejo del computador e internet, de dos instituciones educativas de carácter público y privado, ubicadas en la ciudad y en un municipio de zona rural, que cuentan con salas de informática y conectividad. La información analizada procede de los protocolos automatizados que registran la interacción de los participantes en una actividad inicial de encuesta y las actividades de aprendizaje realizadas en las cuatro unidades de la Revatec descritas en el diseño, y que se generan en foros, wikis y entrevistas (realizadas en un proceso de inducción al manejo de la Red, de interacción entre los participantes y de cierre del proceso).

\section{DISCUSIÓN DE RESULTADOS}

Luego del proceso de análisis de línea por línea de los protocolos de las herramientas comunicativas y colaborativas, se seleccionan un total de 1.257 expresiones completas, las cuales se configuran con la base para la codificación tanto de los procesos de interacción en la Revatec como de la construcción del concepto de tecnología.

Los procesos de interacción se analizan desde tres aspectos: la interacción con la interfaz, con los contenidos y la interacción social, desde la perspectiva que se planteó en los aspectos teóricos. En la interacción con la interfaz se ubican los resultados de ingreso de los estudiantes a la Revatec, en donde se encuentra que el periodo inicialmente contemplado de 8 semanas para el desarrollo de las actividades se extiende por iniciativa de los estudiantes a 16 semanas, por lo cual se opta por incluir también los registros de este tiempo. Durante este tiempo se observa que el $68 \%$ de los participantes registraron entre 11 y 99 ingresos a la red, con un promedio de 512 acciones semanales, lo cual muestra que la interacción con la interfaz está determinada por la culminación de las actividades propuestas. Esto muestra que la participación de los usuarios no es esporádica sino sostenida en el tiempo. 


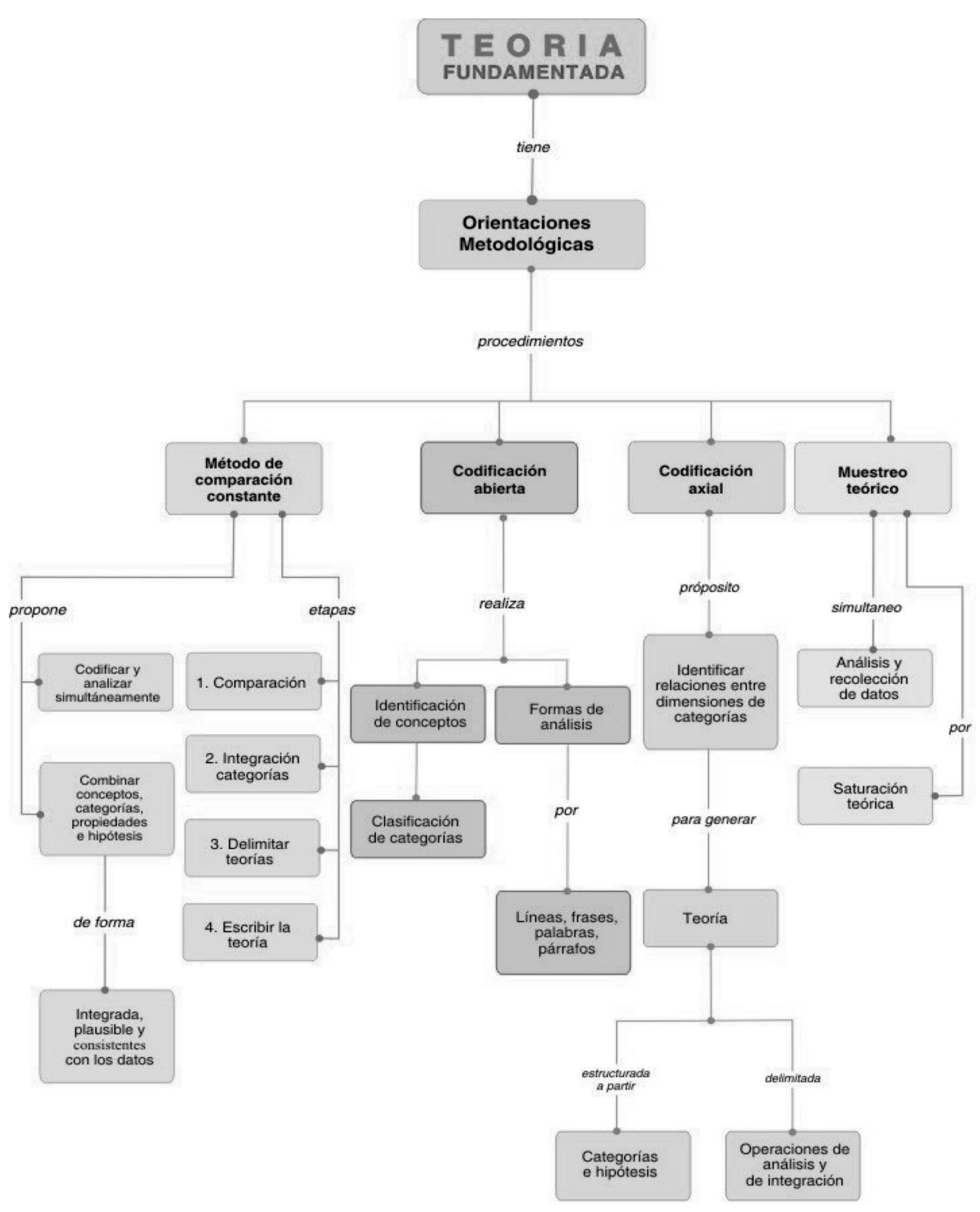

Figura 1. Aspectos metodológicos de la teoría fundamentada

Fuente: Molina (2015, p. 165). 
En la interacción con los contenidos se encuentra que el espacio más visitado es el módulo de interacción, que cuenta con herramientas de comunicación y colaboración como los foros, chat y wikis, seguido del módulo de exploración en los blogs de investigadores y de encuesta inicial, y finalmente el módulo de actividades de aprendizaje compuesto por hipertextos temáticos y mapas conceptuales. Los módulos menos visitados fueron los de consulta e información general, en donde se encuentran la bitácora y la mediateca, como se puede observar en la Figura 2.
El número de aportes promedio realizados por los participantes en la Revatec muestra una participación activa, lo cual se configura, junto con la extensión en el tiempo citada anteriormente, como elemento que indica la conformación efectiva de las RVA (Angulo, Garzón, Mejía, Obando y Posada, 2007; Maldonado y Serrano, 2008; Molina y Briceño, 2010). Se puede observar en la Figura 3 que los espacios colaborativos, como la wiki, presentan un mayor número de aportes de los participantes, seguido por la encuesta inicial y los foros.

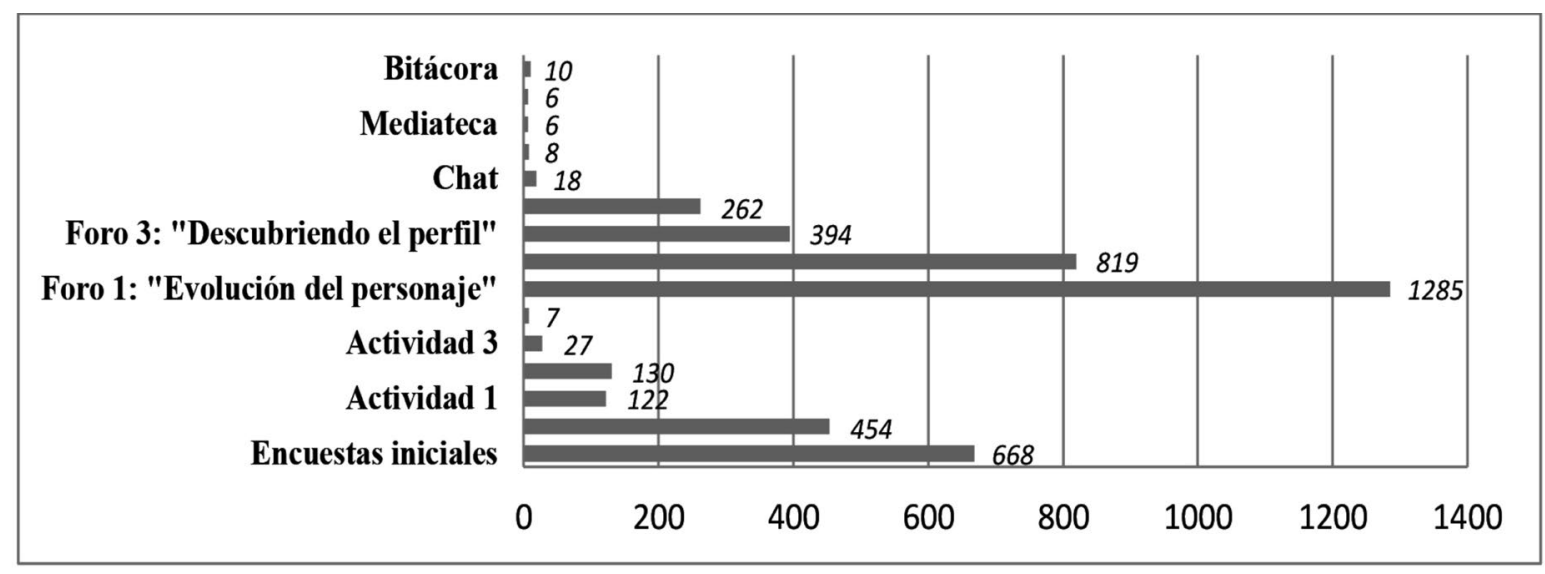

Figura 2. Número de ingresos a los espacios de Revatec.

Fuente: Molina (2015, p. 170).

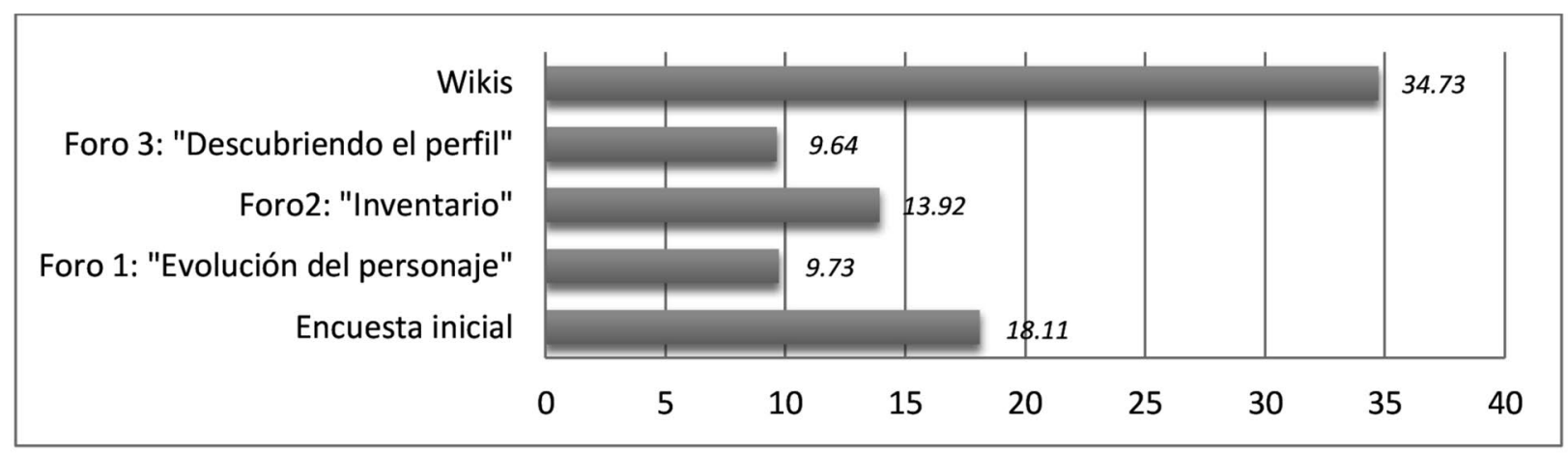

Figura 3. Número de aportes promedio de los participantes en los espacios de Revatec.

Fuente: Molina (2015, p. 172) 
Con respecto a la interacción social, se consideran para este análisis los apartados de los protocolos de las herramientas de comunicación y colaboración, en los que se evidencia un diálogo entre los participantes, es decir, una serie de intercambios comunicacionales: en un sentido, cuando el emisor plantea un aporte y recibe retroalimentación de un receptor; en dos sentidos, cuando el receptor original del mensaje responde al emisor; y multilateral, cuando los mensajes fluyen bilateralmente entre actores que intercambian los papeles de emisor a receptor, sucesivamente.

Se encuentra un total de 32 series comunicativas, de las cuales el 59,4\% presenta un nivel de comunicación en un sentido, ninguna serie presenta comunicación en dos sentidos, mientras que el $40,6 \%$ de las series comunicativas restantes corresponde a una interacción social que cuenta con intercambios multilaterales entre varios participantes. Esto demuestra que en general se encuentra una interacción social incipiente entre los participantes, en comparación con el número de aportes realizados a lo largo del trabajo en la Revatec. Las series comunicativas se orientan a manifestar acuerdo o desacuerdo, argumentar los aportes, agradecer o buscar aprobación, establecer relaciones con otros mediante expresiones de cortesía, agradecimiento y afecto, que evidencian un conocimiento personal previo entre los participantes en el contexto de la institución educativa a la que pertenecen, como se observa en el siguiente ejemplo:

- S11: re bn ¿como la tegnologia a solucionado de comunicación? ... primero fue con señales de humo mucho tiempo des pues se invento la carta, imprenta, telégrafo, el teléfono y por ultimo se an ido in ventado cosas como el computador ipad etc.

- S37: el papel es casi esencial en la vida del hombre dia a dia para escribir y hasta para la mas minima actividad a fasilitado la vida a muchas persona y a dado espacio a muchos mas inventos como el cuaderno que nos sirve parra escribir y anotar lo que se nos olvida.
- S 38: estoy deacuerdo

- S40: muy correcto

- S11: ... Bueno espero que les guste

R/S4: si nicolas estoy de acuerdo contigo!! muah!

(Protocolo actividad 2) ${ }^{3}$.

Se encuentra que las series comunicativas van en aumento a media que se avanza en las actividades de aprendizaje. Se puede evidenciar predominio de retroalimentación, una escasa discusión en los aportes o negociación sobre el significado de la tecnología, en términos de construcción colectiva o colaboración en las interacciones entre los participantes, aunque se observa un aumento al final del trabajo en la red. Esta situación se puede atribuir a que los participantes, quienes no habían utilizado estas herramientas en otro tipo de experiencias, prefieren interactuar con sus compañeros de la misma institución educativa e incluso comentar temáticas de la red en sus encuentros presenciales, razón por la cual no se evidencia en los protocolos de Revatec. Eso se observa en varios hechos: 1) no queda ningún registro de las series comunicativas orientadas a preparar los materiales solicitados ni en parejas o grupos colaborativos; 2) sin embargo, estos materiales y actividades se presentan de manera completa en la red; 3) los registros muestran que el $79,1 \%$ de las actividades se realizan en jornada escolar en horarios de 7 a. $\mathrm{m}$. a 1 p. m. y desde las direcciones de IP correspondientes a las salas de informática de las respectivas instituciones educativas; 4) finalmente, en las entrevistas realizadas se encuentran expresiones como las siguientes:

...Ha si ...también chévere, digamos el foro también fue, fue muy chévere, digamos los comentarios de los otros amigos en el colegio, también lo que habían hecho ellos en la sala de informática para la red... (Protocolo actividad 3).

3 Se transcriben las expresiones de la misma manera como son escritas por los participantes. 
Con respecto a la construcción del concepto de tecnología, luego del proceso de análisis descrito se encuentran cuatro categorías, las cuales se entienden en los términos planteados desde la metodología de la teoría fundamentada, es decir, como denominaciones amplias de términos explicativos más abstractos en los cuales se agrupan los conceptos que comienzan a acumularse y que son identificados en los datos (Strauss y Corbin, 2002). Las características de los conceptos incluidos en cada categoría en las diferentes actividades de aprendizaje en la Revatec se describen en la Tabla 1. La primera categoría se presenta de manera más nutrida en las expresiones de las primeras actividades de aprendizaje aunque disminuye en las actividades finales, con un total de 9 conceptos relacionados y 165 expresiones de los participantes en la Revatec ${ }^{4}$. Esta categoría se refiere a un concepto de tecnología entendido desde los artefactos, materiales e instrumentos que la caracterizan, haciendo reiterada referencia a la utilidad de las máquinas, los procesos para su producción y su función de cara a la satisfacción de necesidades. En esta categoría se menciona que la solución de problemas, la satisfacción de necesidades y los cambios de los artefactos se presentan gracias al avance en los métodos de producción y los procesos de invención, como se observa en los siguientes ejemplos ${ }^{5}$ :

... todo comenso con una roca que luego se convirtio en cuchillos y lanzas y vasigas mas tarde se hicieron catapultas y haci se ha ido tranformando la tecnología... (Protocolo actividad 1)

... A medida que pasa el tiempo las necesidades van aumentando, el hombre necesita mas artefactos para hacer su vida mas sencilla... (Protocolo actividad 2)

4 Es necesario tener en cuenta que algunas de las expresiones pueden tener elementos que hacen alusión a más de un concepto o a más de una categoría.

5 Las expresiones presentadas son un ejemplo y, por supuesto, no son las únicas en las que evidencia la categoría en mención.
La segunda categoría no se presenta de manera nutrida en las primeras actividades, pero posteriormente aparece con mucha más frecuencia hasta convertirse en una categoría con nutridos aportes, con un total de 149 expresiones asociadas a 14 categorías. En esta categoría se conceptualiza la tecnología a partir de aspectos sociales y culturales, haciendo referencia a aquellos artefactos que buscan el bienestar del hombre, su calidad de vida, facilitar el trabajo, la vida cotidiana y la comunicación, creando mejores condiciones y el desarrollo del mundo. Esta categoría se caracteriza por reconocer no solamente que la tecnología tiene un gran impacto en la sociedad y en la conformación de cultura, sino que, a su vez, la sociedad determina el camino que toma la tecnología en sus avances y desarrollos. A continuación se presenta una expresión que ejemplifica lo dicho anteriormente:

< la tecnología ... ha evolucionado porque primero se creia que estaba con el estudio de la destrezas y en esta epoca se ha visto que este personaje puede crear cultura. (Protocolo actividad 2)

...el papel es casi esencial en la vida del hombre dia a dia para escribir y hasta para la mas minima actividad a fasilitado la vida a muchas persona y a dado espacio a muchos mas inventos... (Protocolo actividad 3)

... la sociedad utiliza mucho la tecnología ya casi, por ejemplo las personas todas tiene televisor o un aparato y todas las personas usan eso. Ósea que ya toda la sociedad esta rodea por la tecnología. (Protocolo entrevistas)

Una tercera categoría que emerge de la interacción de los participantes en la red incluye un total de 78 expresiones agrupadas en 7 categorías, que están referidas a entender la tecnología desde los sistemas, conocimientos y procesos que la componen. Se caracteriza por la presencia de artefactos, materiales e instrumentos, de un conjunto de conocimientos y símbolos, de procesos y de personas, como componentes de la tecnología. Estos componentes la caracterizan como una categoría 
compleja en la que el concepto de tecnología interrelaciona los artefactos tecnológicos, los conocimientos que se han generado en torno a ellos y la inteligencia de las personas que han participado en estos procesos, además de la presencia de códigos simbólicos y acuerdos sociales, como se observa en los siguientes aportes:

Lo ha hecho - resolver problemas - por medio de la inteligencia y la tecnologia del hombre y con el conocimiento de todos los productos de la historia....con el conocimiento de cada elemento de acuerdo a la necesidad que requiera el ser humano para la utilidad de cada elemento Tecnologico... (Protocolo actividad 2)

La tecnología es un conjunto de conocimientos que a uno lo puede ayudar a llevar a un fin, todo lo que nosotros podemos hacer, lo que hemos hecho y lo que aprendemos es tecnología y también conocimientos. (Protocolo entrevistas)

Finalmente, la categoría que concibe a la tecnología desde la perspectiva de aplicación de la ciencia es la que se presenta de manera menos reiterada con un concepto y siete expresiones en los aportes de los participantes. Se caracteriza por entender la tecnología como un componente de la ciencia, ya que le debe a esta los conocimientos que sirven de base para el desarrollo de artefactos y procesos tecnológicos. Ello se evidencia en aspectos generales como la presencia de artefactos que se identifican con la ciencia y la participación de científicos en el desarrollo de la tecnología, como un campo de conocimiento relativo al desarrollo de artefactos, a partir del conocimiento científico, lo cual se observa en el siguiente ejemplo:

hay muchos problemas ya que la tecnología soluciona...por ejemplo las enfermedades la tecnología a buscado muchas medicinas para curarlas... los problemas sean solucionado gracias a la evolución del ser humano de la maquinaria y la evolución de la ciencia. (Protocolo actividad 3)
Las expresiones encontradas muestran la presencia simultánea de cuatro categorías, lo que puede indicar una construcción conceptual con elementos diversos que se integran y reflejan el desarrollo de elementos cognitivos orientados a la solución de problemas tecnológicos como respuesta a una necesidad del hombre, a un vacío de conocimiento o simplemente gracias a la curiosidad, lo que provoca el desarrollo de procesos encaminados a plantear soluciones, que se caracterizan por mejorar los artefactos tecnológicos, innovarlos o crear otros, mediante unos métodos que no se especifican en los aportes de los participantes en la red. Esto implica contar con unos fines claros y con la combinación de elementos investigativos y prácticos, que permiten llegar a una solución. Las categorías halladas y el proceso de interacción que lleva a la construcción del concepto de tecnología muestran una suerte de transformación dinámica del concepto en los participantes de Revatec, en la medida en que se desarrollan las actividades de aprendizaje propuestas.

Se encuentra que a pesar de que la red plantea un proceso secuencial de actividades, la construcción del concepto no lo es, es decir, en el desarrollo de cada uno de los momentos de interacción en la Revatec no se encuentra un orden invariante en la manera como surgen estas categorías o en la cantidad de expresiones que se registran en cada una de ellas. Las expresiones no muestran ningún tipo de continuidad en la manera como emergen en el proceso de construcción conceptual, pues las cuatro categorías halladas se presentan desde el primer momento de interacción en Revatec, y aunque algunas tienen más códigos que otras, sus características son claras desde las primeras expresiones analizadas, lo que se evidencia en el planteamiento de conceptos iniciales, de categorías y de relaciones entre ellas, tal como lo plantea el proceso de análisis desde la teoría fundamentada.

El proceso de construcción no es lineal o uniforme, por el contrario, se encuentra discontinuo, sinuoso; no solo presenta dos dimensiones en las 
que se encuentran las categorías y los conceptos al interior de cada una de ellas, sino que muestra rastros de una tercera dimensión en la cual se puede evidenciar la profundidad conceptual que se caracteriza por la riqueza de las relaciones que se establecen entre estos conceptos y por tanto entre las categorías halladas. Desde esta perspectiva, se detectan tres formas de organización o dimensiones en el análisis realizado a partir de categorías, campos conceptuales y relaciones. La primera corresponde a las categorías emergentes sobre lo que se entiende por tecnología. La segunda, a los campos conceptuales que permiten comprender las características de cada una de las categorías y son transversales a todas ellas, como por ejemplo el tipo de artefactos que caracterizan la categoría, el tipo de necesidad que atiende, los problemas que soluciona y las relaciones entre sociedad y tecnología. En la tercera forma de organización se presentan aquellos elementos diferenciales que permiten reconocer las características particulares en cada uno de los conceptos y categorías, establecer unos límites claros entre ellos y la complejidad que se presenta en el proceso.

\section{CONCLUSIONES}

Los resultados de este estudio permiten concluir que en términos de interacción con la interfaz y los contenidos de la RVA, hay tendencia de carácter operativo pragmático de los participantes en la red, que se caracteriza por la inclinación a realizar acciones tendientes a ser visibles, estar "activos" al interior de la red y realizar aportes, por encima de otras actividades como leer o analizar, que son propias de la interacción con contenidos hipermediales, de naturaleza más individual. Con respecto a la interacción social, se observa una tendencia a aumentar las series comunicativas de manera sostenida desde la primera hasta la última actividad de aprendizaje, incrementando los procesos de retroalimentación en un sentido, hasta llegar a intercambios multilaterales de diálogo entre varios participantes, quienes exponen sus niveles de acuerdo, desacuerdo, hacen críticas al trabajo de otros grupos y argumentan sus posiciones de una manera más firme. Al final del trabajo en la red, se observa el intercambio comunicativo multidireccional y la negociación comprensiva e interpretativa de significados a partir de los contenidos propuestos en las actividades de aprendizaje, lo que implica la selección, exploración, consecución-retroalimentación y retorno, que se presenta en los diferentes aportes de los participantes. Sin embargo, sería necesario explorar en procesos posteriores de indagación, la manera como estas series comunicativas aportan a procesos colaborativos de construcción conceptual.

De otra parte, se concluye que la construcción del concepto de tecnología no se lleva a cabo de manera progresiva, siguiendo algunas de las etapas previstas. Sobresale en este proceso la baja influencia que tienen las actividades de carácter colaborativo en la red y que permite mostrar cómo, desde las interacciones iniciales de los jóvenes, se evidencian categorías que conciben la tecnología como artefactos, materiales e instrumentos; como elemento social y cultural; como sistemas de conocimientos y procesos; y como ciencia aplicada. El proceso de interacción y construcción conceptual de los participantes permite vislumbrar que efectivamente la RVA se configura como mediadora en la construcción conceptual por parte de los adolescentes y jóvenes registrados en la red.

A partir de aquí bien podría pensarse que la incursión de las RVA en los procesos formales de construcción conceptual puede aportar elementos interesantes no solamente en la mediación didáctica, sino también en relación con la conceptualización de la tecnología, en la configuración de un cuerpo de conocimiento propio, que muestra una tendencia hacia la integración y complementariedad de enfoques. Se puede vislumbrar desde esta experiencia que se está gestando un cambio en la manera como las nuevas generaciones empiezan a concebir la tecnología como un concepto metadisciplinar, complejo y multidimensional que permite articular e interrelacionar las categorías 
encontradas de forma interdependiente y complementaria. Esto evidencia que se supera, entre otros aspectos, la mirada simplificada, reduccionista, unidimensional o disyuntiva de los saberes, adoptando un criterio amplio que conjuga diferentes enfoques. De otro lado, minimiza la dicotomía entre lo instrumental y lo conceptual, entre lo social y la aplicación de la ciencia, entre los procesos reflexivos y sistémicos, para solucionar problemas sin separarlos de su contexto y comprender los sistemas desde sus elementos sociotécnicos sin separarlos de su entorno. Esto implica que el concepto de tecnología estaría llamado a tender puentes con otro tipo de conocimientos, a establecer conexiones con otras dimensiones, de tal manera que se transforme en un eje integrador, articulador y orientador de los conocimientos.

Finalmente, es necesario plantear una reflexión en torno a la implementación de esta experiencia en los contextos escolares y los aspectos derivados de los resultados de este estudio, en términos de entender la propuesta de los contenidos de la Revatec, como unidades de trabajo académico, que bien pueden incorporarse en los procesos de formación. Por su parte, los resultados pueden dar pie a una nueva perspectiva para los maestros sobre la forma como los estudiantes realizan la construcción de conceptos, de tal manera que se puedan generar estrategias pedagógicas y didácticas coherentes con la construcción conceptual.

Los resultados de este estudio se orientan a fortalecer una temática de trabajo poco explorada en el área de la educación en tecnología, dado que permite explorar otra maneras como los estudiantes interactúan en RVA

\section{RECONOCIMIENTOS}

Este artículo presenta los resultados de la tesis doctoral laureada que lleva el mismo título, "Construcción del concepto de tecnología en una red virtual de aprendizaje" ${ }^{\prime \prime}$, realizada en el Doctorado Interinstitucional en Educación (DIE), de la Universidad Distrital Francisco José de Caldas, en 2015.

\section{REFERENCIAS}

Angulo, F., Garzón, D., O., P., Mejía, M., Obando, G., y Posada, F. (2007). Aspectos Teóricos y Metodológicos para la Consolidación de una Red de Aprendizaje desde la Didáctica de las Matemáticas. Colciencias-Universidad del Valle-Universidad de Antioquia. Cali: Colciencias-Universidad del Valle-Universidad de Antioquia.

Ausubel, D., Novak, J., y Henesian, H. (1983). Psicología educativa: un punto de vista cognoscitivo. México: Trillas.

Barbera, E., Badia, A., y y Mominó, J. (2001). La incógnita de la educación a distancia. Barcelona: ICE-Horsori.

Benassini, C. (2003). De las comunidades sociales a las ¿comunidades? virtuales. Disponible en http:// www.comminit.com/la/lasc/sld-4423.html(Consultado el 30 de mayo de 2012).

Bonilla, M., Molina, R., Martínez, L. y Narváez, D. (2011). Formación de docentes e investigación en redes virtuales de aprendizaje. Bogotá: Universidad Distrital Francisco José de Caldas.

Bossolasco, M. (2010). El foro de discusión: entorno mediado para mediación cognitiva. Mendoza: Editorial Virtual Argentina.

Briceño, S., Lugo, R., Molina, R., y Munevar, P. (2006). Modelo curricular en informática educativa para el Distrito Capital. Bogotá: Uniminuto - Secretaría de Educación del Distrito.

Broncano, F. (2000). Mundos artificiales: Filosofía del cambio tecnológico. México: Paidós .

Cajas, F. (2001). Alfabetización científica y tecnológica: la transposición didáctica del conocimiento tecnológico. Enseñanza de las Ciencias, 19, 243-254.

Charmaz, K. C. (2000). Constructing Grounded Theory: Practical Guide through Qualitative Analysis. Conoma State University: SAGE Publications Ltd.

Clarke, A. (2005). Situational Analysis Grounded Theory After the Postmodern Turn. San Francisco: Universidad de California .

Cole, M. (1999). Psicología cultural. Madrid: Ediciones Morata. 
Creafuture. (2010). Informe Teens: Cómo son los adolescentes y cómo cambian sus hábitos de consumo. Creafuture. Disponible en http://www.slideshare.net/ amcuadrado/evolucion-hbitos-de-consumo-adolescentes-2010 (Consultado el 30 de mayo de 2012).

Creswell, J. (2005). Educational research: Planning, conducting, and evaluating quantitative and qualitative research. Upper Saddle River: Pearson Education.

Cupani, A. (2006). La peculiaridad del conocimiento tecnológico. Scient y Studia, 3(4), 353-371.

Dillenbourg, P. B. (1996). The evolution of research on collaborative learning. En E. S. Reiman (Ed.), Learning in Humans and Machine (pp. 189- 211).

De Kerckhove, D. (1999). Inteligencias en conexión. Hacia una sociedad de la Web. Barcelona: Editorial Gedisa.

García, L. (2001). La educación a distancia. Barcelona: Editorial Ariel.

Hakkarainen, K., Palonen, T., Paavola, S., y Lehtinen, E. (2004). Communities of networked expertise: Professional and educational perspectives. Amsterdam: Elsevier.

Gros, B. (2004). La construcción del conocimiento en la red: límites y posibilidades. E. U. Salamanca (Ed.). Disponible en http://dialnet.unirioja.es/servlet/oaiar$\mathrm{t}$ ?codigo $=1048854$. (Consultado el 22 de septiembre de 2009).

Harasim, L., Starr, R., Murria, T., y Teles, L. (2000). Redes de aprendizaje: guía para la enseñanza y el aprendizaje en red. Barcelona: Editorial Gedisa.

Heidegger, M. (1994). La pregunta por la técnica: conferencias y artículos. Barcelona: Odos.

Hillman, D., Wills, D., y Gunawardena, C. (1994). Learner interface interaction in distance educativo: an extension of contemporary models and strategies form practitioners. Ther American Journal of Distance Education, 8, 30-42.

Hernández, R. (2008). Metodología de la investigación. México: McGrawHill

Hronzsky, I. (2001). Algunas observaciones sobre la reciente filosofía de la tecnología en Europa: el caso de Alemania . En J. López, J. Lujan, y E. García (Eds.), Filosofía de la Tecnología (pp. 107-123 ). Madrid: OEI.
Hughes, T. (1983). Networks of Power. [En línea]. The Johns Hopkins University Press. Disponible en http://www.nyu.edu/projects/nissenbaum/papers/Networksofpower.pdf (Consultado el 2 de agosto de 2013)

Inciarte, A. (enero de 2011). Generación de teoría fundamentada. [En línea]. Universidad de Zulia. Disponible en http://www.eduneg.net/generaciondeteoria/files/INFORME-TEORIA-FUNDAMENTADA.pdf (Consultado el 2 de agosto de 2011).

International Society for Technology in Education (Iste). (2007). National Educational Technology Standards for Students: The Next Generation. Disponible en www.iste.org (Consultado el 15 de septiembre de 2009, de Iste)

Johnson, D., y Johnson, R. .. (1998). Cooperative Learning, Values, and Culturally Plural Classrooms. Cooperative Learning Center at the University of Minnesota. Disponible en http://www.clcrc.com/ pages/CLandD.html (Consultado el 5 de mayo de 2007)

Lévy, P. (2007). Cibercultura: la cultura de la sociedad digital. Barcelona: Ediciones Arthropos.

Latour, B. (2005). Reassembling the Social, An Introduction to Actor-Network- Theory. Oxford University Press, Inc. Disponible en http://dss-edit.com/plu/ Latour_Reassembling.pdf (Consultado el 8 de febrero de 2013)

Maldonado, L., y Serrano, E. (2008). Construcción de una red de aprendizaje. Revista Nómadas, 29, 211-222.

Medina, M. (2003). La cultura de la tecnociencia. En C. S. Bueno, y C. B. Santos (Ed.), Nueva tecnologías y cultura (pp. 29-74.). Barcelona: Anthropos Editorial.

Marqués, P. (2001). Evaluación y selección de software educativo. Disponible en http://www.tecnoneet. org/docs/2002/62002.pdf (Consultado el 5 de mayo de 2012).

Mertens, D. (2005). Research and Evaluation in Education and Psychology: Integrating diversity with quantitative, qualitative, and mixed methods. Boston: Sage. 
Ministerio de Educación Nacional. (2008). Ser competente en tecnología: una necesidad para el desarrollo. Bogotá.

Mitcham, C. (1998). ¿Qué es la filosofía de la tecnología? Barcelona: Editorial Arthropos.

Molina, R., y Briceño, S. (2009). Estrategias metodológicas para la consolidación de redes virtuales de aprendizaje. Bogotá: Universidad Distrital Francisco José de Caldas.

Molina, R., y Briceño, S. (2010). Redes virtuales de aprendizaje: una experiencia de formación con docentes. Bogotá: Universidad Distrital Francisco José de Caldas.

Mumford, L. (1971). Técnica y civilización. Madrid: Alianza Editorial.

Osorio, C. (2003). Aproximaciones a la tecnología desde los enfoques en CTS. Disponible en http://www. oei.es/salactsi/osorio5.htm (Consultado el 2 de febrero de 2011).

Osorio, C. (2007). El determinismo tecnológico: una reflexión crítica de la teoría de los sistemas tecnológicos. Universidad de Oviedo.

Palloff, R., y Pratt, K. (2005). Collaborating online. Learning together in community. San Francisco: Jossey - Bass a Wiley Imprint.

Piaget, J. (1972). El Lenguaje y el pensamiento en el niño. Buenos Aires: Guadalupe.

Pinch, T. (1997). La construcción social de la tecnología: una revisión. En M. Santos, y R. Díaz (Eds.), Innovación tecnológica y procesos culturales: Nuevas perspectiva teóricas (pp. 20-38). México: Fondo de Cultura Económica.

Pinch, T., y Bijker, W. (1984). The social construction of facts and artifacts: Or how the sociology of science and the sociology of technology might benefit each other. Disponible en http://www.ihs.uw.edu. $\mathrm{pl} / \mathrm{wp}$-content/uploads/2012/10/The-Social-Construction-of-Facts-and-Artefacts.pdf (Consultado el 16 de agosto de 2013).
Quintanilla, M. (1993-1994). Seis conferencias sobre filosofía de la tecnología. Revista Plural, 11-12.

Rapp, F. (1981). Filosofía analítica de la técnica. Barcelona: Editorial Alfa.

Rengil, L. (2002). Estrategias docentes para educar con nuevas tecnologías de la comunicación. Mazatlán: Segundo Congreso de Imagen y Pedagogía.

Rueda, R. (2006). Tecnologías de la información y la comunicación - Informática educativa en la formación inicial de maestros en Bogotá. Bogotá: IESCO - SED.

Scolari, C. (2004). Hacer clic: hacia una sociosemiótica de las interacciones digitales. Barcelona: Editorial Gedisa.

Strauss, A. (1987). Qualitative analysis forsocial scientists. USA: Cambridge University Press.

Strauss, A., y Corbin, J. (2002). Bases de la investigación cualitativa. Técnicas y procedimientos para desarrollar la teoría fundamentada. (E. Zimmerman, Trad.) Medellín: Editorial Universidad de Antioquia.

Unigarro, M., Castaño, L., Mestre, G., Molina, R., Prado, M., Ruiz, E., y otros. (2003). Las comunidades virtuales de aprendizaje en el contexto de las redes universitarias. Universidad Autónoma de Occidente. Disponible en http://sev.cuao.edu.co/ pages/docs/comuvirt_aprendizaje_universitarias. pdf (Consultado el 9 de enero de 2009).

Vygotski, L. (1934). Pensamiento y lenguaje. Buenos Aires: Editorial La Pléyade.

Williams, P. S. (2007). Fundamentos del diseño tecnopedagógico en e-learning: Modelos de diseño instruccional. Barcelona: Universidad Oberta de Catalunya.

Winner, L. (1985). ¿Tienen política los artefactos? Disponible en http://www.oei.es/salactsi/winner.htm (Consultado el 9 de enero de 2012). 